\title{
Visual detection in relation to display size and redundancy of critical elements'
}

\author{
W. K. ESTES AND H, A. TAYLOR \\ STANFORD UNIVERSITY
}

Visual detection was studied in relation to displays of discrete elements, randomly selected consonant letters, distributed in random subsets of cells of a matrix, the $S$ being required on each trial to indicate only which member of a predesignated pair of critical elements was present in a given display. Experimental variables were number of elements per display and number of redundant critical elements per display. Estimates of the number of elements effectively processed by a $S$ during a $50 \mathrm{~ms}$. exposure increased with display size, but not in the manner that would be expected if the $S$ sampled a fixed proportion of the elements present in a display of given area. Test-retest data indicated substantial correlations over long intervals of time in the particular elements sampled by a $S$ from a particular display. Efficiencies of detection with redundant critical elements were very close to those expected on the hypothesis of constant sample size over trials for any given display size and were relatively invariant with respect to distance between critical elements.

In a previous article (Estes \& Taylor, 1964) we have presented a detection method for assessing the amount of information assimilated by an observer from a tachistoscopically presented visual display. In many essentials the procedure corresponds to that of a two alternative, forced-choice signal detection experiment. On each trial the $S$ is shown for a short interval, $50 \mathrm{msec}$. in the previous study, a display containing a number of symbols. Two symbols are designated as critical elements and the rest as noise elements, each display containing exactly 1 of the 2 critical elements. The S's task on each trial is simply to observe the display and indicate which of the 2 critical elements he believes to have been present.

One of our purposes in developing this technique was to provide estimates of the number of elements perceived from a brief display with minimal perturbation by retention loss. This procedure may be more sensitive than either the sampling method of Sperling (1960) or the indicator method of Averbach \& Coriell (1961). With the detection method there is minimal retention loss during the time required for the $S$ to give his response and the visual field immediately following a display is not disturbed by the appearance of an indicator mark. A question of considerable theoretical interest which arises in connection with comparisons of these various techniques for assessing perceptual span is that of whether the perceptual response which a s must make to an element of a display in order to categorize it as signal or noise is the same as the response required to permit the $S$ to designate the symbol by name in a subsequent report. While recognizing that the alternative methods for assessing perceptual span cannot be satisfactorily compared for sensitivity until this question is answered, we defer fur- ther consideration of it until the behavior involved in the detection task has been studied in greater detail.

The two experiments to be reported in this paper were designed to provide information bearing on alternative theoretical assumptions regarding the process of sampling the elements in a display. From results of our preliminary experimentation (Estes \& Taylor, 1964), it is clear that with displays of the order of 5 to 10 elements (random samples of consonants printed in linear arrays) the average number of elements per display effectively processed by adult Ss is appreciably greater than that revealed by the classical verbal report procedure with similar materials, but still significantly less than should be possible on the basis of psychophysical considerations alone. Under our conditions, a display of up to 10 to 12 elements should be expected to generate an image falling well within the area of clear vision on the retina. With substantially longer exposure times, an observer has no difficulty in reporting all of the elements of one of these displays while fixating on a point in the center, and consequently there would seem to be no explanation in terms of the visual system for the fact that our Ss were able to use information from no more than about $70 \%$ of the displayed elements during $50 \mathrm{msec}$. exposures. The simplest explanation which comes to mind, to the effect that the amount of information utilizable from a display is restricted by a fixed upper limit on the number of elements which can be registered in a temporary memory storage of some sort, appears to be excluded by the forms of the functions obtained in our previous study relating estimates of elements perceived to size of the display. These functions were, however, quite compatible with predictions from a serial processing model, which will be discussed in more detail below. The first of the two experiments to be reported was planned to obtain more information on the process responsible for the upper limit on the amount of information obtainable from a display of a given size; it deals with probability of correct detection as a function of display size under conditions differing in some important respects from those of the preceding study. The second experiment has to do with the independence of response to different elements of a display, the experimental variable of principal interest being the introduction of redundant critical elements.

\section{Experiment 1}

\section{Defection as a function of Number of Elements in Displays of} Constant Area

On the basis of much accumulating data concerning information processing by human Ss (see Broadbent, 
1958), we believe it essential to assume that at some point in the S's response system the elements of a tachistoscopic display must be reacted to singly; figuratively speaking, the elements, though initially registered in parallel on the retina, must pass at some point through a channel which permits passage of only one element at a time. The serial processing model, proposed in our earlier paper, has this characteristic. The principal assumptions of the model are:

(1) When a display containing $\mathrm{D}$ elements is exposed for a short interval, a subset of elements is registered in the receptor apparatus. In the present study we have chosen experimental conditions such that we should expect all of the elements in the display to be so registered.

(2) Following the exposure, the traces of the display in the nervous system fade exponentially in such a way that, during each successive interval of time $\Delta t$ (taken just long enough for scanning of a single element) following the display, there is some fixed probability $s$ that the traces of the display will have passed below the threshold level at which they can influence behavior.

(3) During and following the display, until the stimulus traces pass below threshold, the registered elements are scanned one at a time. As each element is scanned, it is classified either as signal or as noise; and if the former, it leads to a report as to the critical element detected.

(4) If the stimulus traces fade below threshold before the critical element is scanned, the $S$ gives a report at random.

The probability of scanning any number $\mathrm{k}$ of elements from a display is then given by

$$
P_{k}= \begin{cases}0 & \text { if } k<m \\ s(1-s)^{k-m} & \text { if } m<k<D \\ (1-s)^{D-m} & \text { if } k=D\end{cases}
$$

where $\mathrm{m}$, the number of elements scanned during the stimulus exposure, is assumed to be a linear function of exposure time.

According to this formulation the mean number of elements effectively processed is expected to be an increasing function of display size simply because, up to the limiting number of elements that can be registered on the retina, the larger the display the larger is the possible number of elements scanned on a trial.

A principal alternative interpretation to that of the scanning model, which is in some respects more parsimonious, is the following, which might be termed a differential perceptibility model. It is well known that when a S fixates his gaze on the central point of a display area, such as that of our tachistoscope, there exists a probability distribution over the area of the potential display such that elements appearing at the center have highest probabilities of being seen clearly, the probabilities falling off in all directions toward the periphery. Assuming that this probability-of-seeing distribution is determined by the conditions of area, brightness, and exposure duration of a given experiment, we might suppose that in our previous study conditions were such that for the smallest displays nearly all of the elements fell in the central area of high probability but that with increasing display size the added elements fell in the peripheral areas, thus increasing the total mean number of elements seen per display. Since, in that study, the elements were presented in linear arrays with constant distances between elements, and the center of each display was at the fixation point, this interpretation could account nicely for the results obtained.

In order to obtain evidence which might be more useful in deciding between the two types of interpretation mentioned above, the displays were prepared according to a different principle in the present study, the plan being to vary number of elements per display while holding the area over which these elements were spread approximately constant. The plan followed was to embed each display, regardless of size, in a 4 by 4 matrix approximately 1 in square. For the largest display used, 16 elements, the 16 cells of the matrix were simply filled by a random sample of 16 consonant letters. For the smallest display size, 8 elements, half of the cells were filled, but with the restriction that each row and each column of the hypothetical matrix contain 2 elements; in the intermediate display size, 12 elements, the matrix was filled similarly, but with the restriction that each row and each column contain exactly 3 elements. Thus the vertical and horizontal distances from the fixation point spanned by the elements of a display were constant while the number of elements per display varied from 8 to 16 . Now, according to the interpretation of the display size function in terms of differential perceptibility, the number of elements perceived per display should on the average be a constant proportion of the number of elements presented in the display. According to the serial processing model, on the other hand, the mean number of elements effectively processed should increase as a function of display size, but the proportion should decrease in a negatively accelerated fashion with linear increases in display size.

\section{Method}

Apparatus. The front of the tachistoscope ${ }^{2}$ appeared to $S$ as a wooden panel 17-1/2 in square surrounded by $a$ dark hood 8 in deep. Centered in the panel at a height of $9-1 / 2$ in from the bottom was a window of half-silvered glass, 2 in high by $5-1 / 2$ in wide. Below this was a sloping black shelf, extending from the panel to the edge of the hood, such that its reflection rather than those of objects in the experimental room appeared in the glass.

Stimulus exposure was effected by turning on a coldcathode fluorescent tube in the left-hand (from S's point of view) of two equal sized compartments behind the window; this light provided even illumination of the stimulus display which was positioned $2-1 / 2$ in behind the window. The light was controlled by means of an external control, power supply, and waveform generator $^{3}$. In the present experiments the stimulus presentation interval was always $50 \mathrm{msec}$ (.05 sec.). 
The S was allowed to hold his head at that distance from the window at which he could most comfortably read sample material, but in no case closer than 8 in to the window. A fixation point was provided in the form of a small round spot of white paper positioned by each $S$ on the sloping shelf below the window so that its reflection, seen only while the exposure light was not on, appeared on the center position of a sample display.

The stimulus field reflected $14.5 \mathrm{ft}$-lamberts during exposures and less than .1 ft-lambert between exposures, as cletermined with a Macbeth illuminometer.

Stimulus materials. The stimulus materials were arrays of consonant letters typed, by means of an IBM electric typewriter, in large capitals on white cards in the following manner: Potential 16-cell matrices, 4 letters wide and 4 letters high, were filled with either 8 , 12, or 16 letters. The size of the type used and the spacing were such that each display was 1 in high and $7 / 8$ in wide.

For each display size, D, a complete set of displays consisted of 32 cards. These were two parallel sets of 16 which differed only in the signal,i.e., the member of the critical letter pair, $B$ and $F$, which was present. Each critical letter appeared once in each cell of the 4 by 4 matrix, always accompanied by the same D-1 noise letters. For a given display size, the set of noise elements was drawn randomly without replacement from the set of 18 available consonants. The matrices were filled randomly within the severe constraints imposed by the foregoing rule and a rule that each row and each column contain the same number of letters. Thus at $\mathrm{D}=8$ each row and column contained 2 letters; at $\mathrm{D}=12$ each row and column contained 3 letters, and at $D=16$ each row and column contained 4 letters. In this way the area on the card covered by the matrices was independent of the number of letters, while the density of letters in this area increased with increasing $D$. These stimulus materials were typed in the IBM Executive Directory style, 14 type, code $66, \mathrm{pk}$, type-style.

Procedure. Ss were 20 Stanford students, paid for participation. The experimental room was illuminated by a $150-w$ bulb encased in a white globe mounted on the ceiling; this was adjusted to about $1 / 3$ normal brightness by means of a wall dimmer switch.

After a $5 \mathrm{~min}$. allowance for adaptation to room brightness, each $S$ was seated at the apparatus and given a sample display with which to find a comfortable observing position and then to adjust the fixation spot. He was then instructed to report whether each display contained a " $\mathrm{B}$ " or an " $\mathrm{F}$ ", guessing whenever unsure. The 8letter cards were now exposed, in a different random order for each S. A new stimulus exposure followed the response to the previous display by about $2 \mathrm{sec}$. and was preceded by $1 / 2 \mathrm{sec}$. by a $5 \mathrm{msec}$. flash of the exposure light to ensure alertness.

When the 8-letter cards had all been exposed, the 12-letter displays were then shown, then the 16-letter displays. At the conclusion of this series $\mathrm{S}$ was given a 5 min. rest while the cards were reshuffled; they were
TABLE I Statistics for Proportions of Correct Detections from Displays with Single Critical Elements (Data Pooled over 20 Subjects and 3 Replications)

\begin{tabular}{lccl} 
& \multicolumn{3}{c}{ Display Size } \\
& 8 & 12 & 16 \\
\hline Mean & .777 & .722 & .673 \\
S.D. & .076 & .073 & .062 \\
S.E.M & .017 & .016 & .014 \\
\hline
\end{tabular}

then presented again in the same fashion, reshuffled, and presented once more. Thus a total of $3 \times 32=96$ determinations was obtained for each display size. Results

Statistics for the 20 Ss pooled over the three replications at each display size are given in Table 1. About all that can be concluded from these data is that, as would be anticipated, proportion of correct detections decreased significantly with increasing display size, and that there was substantial variability among Ss in efficiency of detection. If there were no individual differences, and if trials were independent, the standard deviations in Table 1 should have run from approximately .042 at $\mathrm{D}=8$ to .048 at $\mathrm{D}=16$. The tendency for standard deviations to approach the standard deviation of the appropriate binomial distribution as $\mathrm{D}$ increases is to be expected, for with increasing display sizes increasing proportions of the obtained successes must be achieved by guessing and the probability of success by guessing is the same for all Ss.

For most theoretical purposes it is desirable to convert the raw data in terms of proportions of correct responses into estimates of numbers of elements effectively processed at the various display sizes. To do this, we proceed as in our previous study, correcting for successes achievable by guessing according to the formula

$$
P C=\frac{P}{D}+\left(1-\frac{P}{D}\right) \frac{1}{2}
$$

where $\mathrm{p}_{\mathrm{C}}$ represents probability of a correct response, $P$ the number of elements effectively processed, and D the number of elements in the display. The basis of this relation is that, since the critical elements are randomly placed in the displays, the probability that the critical element falls among the $P$ elements perceived is $P / D$; and whenever the critical element does not fall among those perceived the probability of a correct response is $1 / 2$ since both critical elements occurred equally often in random sequence. Replacing $p_{C}$ by the observed proportion of correct detections at a given display size, we can solve the equation above for $P$ and thus obtain an estimate of this theoretical quantity in terms of observables:

$$
\hat{P}=(2 p-1) D
$$

Using this procedure, the estimates of mean number of elements processed per display have been computed for each of the three tests given on each display size 
TABLE II Estimates of Mean Number of Elements Processed for each Display Size

\begin{tabular}{|c|c|c|c|c|c|c|}
\hline \multirow[b]{2}{*}{ Test } & \multicolumn{3}{|c|}{$\begin{array}{c}\text { Observed } \\
\text { Display Size }\end{array}$} & \multicolumn{2}{|c|}{$\begin{array}{l}\text { Theoretical } \\
\text { Display Size }\end{array}$} & \multirow[b]{2}{*}{16} \\
\hline & 8 & 12 & 16 & 8 & 12 & \\
\hline $\mathrm{T}_{1}$ & 3.82 & 5.09 & 4. 10 & 3.88 & 4.50 & 4.80 \\
\hline $\mathrm{T}_{2}$ & 4.72 & 5.20 & 6.24 & 4.55 & 5.53 & 6.10 \\
\hline$T_{3}$ & 4.58 & 5.81 & 5.82 & 4.58 & 5.56 & 6.13 \\
\hline Pooled & 4.43 & 5.33 & 5.57 & 4.36 & 5.22 & 5.70 \\
\hline
\end{tabular}

and these, together with estimates averaged over the three tests, are given in the left-hand three columns of Table 2. Two conclusions immediately emerge from consideration of these values. Firstly, except perhaps for the first test, $T_{1}$, given before the Ss had reached their limit of improvement as a function of practice, the mean number of elements processed increased as a function of display size. For the pooled data, a signed ranks test shows the increases from $D=8$ to $D=12$ and from $D=8$ to $D=16$ to be significant at the .02 level; the differences between values for $D=12$ and $D=16$ were not significant. However, the increases in number of elements processed are far from proportional to increases in display size as D goes from 8 to 12 to 16 ; consequently there would seem to be no support for the hypothesis that the dependence of detection rate on display size can be accounted for entirely, or even primarily, in terms of the factor of differential perceptibility. The parameter $s$ of the serial processing model has been estimated from the data by least squares and theoretical predictions for mean numbers of elements processed at each display size are given in the right-hand side of Table 2. For the second and third tests and for the pooled data, these predicted values exhibit reasonably satisfactory correspondence with the observed data.

Since each $\mathrm{S}$ was tested on each display three times at widely spaced intervals, data on changes of response to a given display over successive tests may be expected to provide information concerning the scanning process. If the order in which the elements of a display are scanned were entirely random, then the probability of a correct response to a given display on a later test should be the same regardless of whether's's response to that display on an earlier test was correct or incorrect. If, on the other hand, scanning follows a relatively fixed path, for example beginning at the fixation point and proceeding outward in some orderly fashion, then correct detection to a given display on an earlier test will tend to be followed by correct detections on later tests and failures on early tests by failures on later tests. Probability of a correct detection on a later test following a correct detection on an earlier test should, then, be greater than the overall probability of a correct detection. However the probability of correct given correct should be less than unity for two reasons: Firstly, some successes will occur by chance and these will be followed by successes on later tests on the same displays only to the extent that chance would allow; second- ly, even though the "scanning path" were precisely the same from trial to trial on a given display, there would still, according to the serial processing model, be variation in the distance travelled along this path. Also, it is a consequence of the assumptions of the serial processing model that, for the parameter values of this study, the divergence between probability of correct given correct and probability of correct given incorrect should increase as display size increases. It can be shown that, in general, the difference between probability of correct given correct and probability of correct given incorrect should start at 0 for the smallest displays, increase to a maximum and then decrease to 0 as display size increases. For the particular parameter values of this experiment, the differences should be smallest at $D=8$, increase considerably at $D=12$, and increase only slightly further as $D$ goes from 12 to 16 . On the whole, the pattern exhibited in Table 3 appears to bear out expectations on the basis of our assumptions. At the very least, it can be concluded with confidence that there is substantial positive correlation over successive tests in the particular elements processed by a $\mathrm{S}$ from a given display even when the tests are separated by long time intervals and much interpolated activity.

The fixed sample size model can account for some aspects of the test-retest data but apparently cannot accommodate the pattern of quantitative relationships shown in Table 3. Assuming that the subject draws a sample of $d$ elements from a display of size $D$, with the samples on any two successive tests having a subset of $\mathrm{d}_{\mathrm{O}}$ elements in common, the model yields the following theoretical functions

and

$$
\begin{aligned}
P\left(C_{i}: C_{i}\right) & =\frac{1}{4}\left(1+\frac{2 d}{D}+\frac{d o}{D}\right) / \frac{1}{2}\left(1+\frac{d}{D}\right) \\
& =\frac{D+2 d+d o}{2(D+d)},
\end{aligned}
$$

$$
\begin{aligned}
P\left(C_{i}:\left.\right|_{j}\right) & =\frac{1}{4}\left(1-\frac{d o}{D}\right) / \frac{1}{2}\left(1-\frac{d}{D}\right) \\
& =\frac{D-d O}{2(D-d)},
\end{aligned}
$$

the notation being the same as that of Table 3. Taking $\mathrm{d}=4$ for the 8-element display, on the basis of the data of Table 2, and estimating $\frac{\mathrm{do}}{\mathrm{D}}=.34$ from the pooled $\mathrm{I}_{2} \mathrm{C}_{3}$ data, we obtain the theoretical values $P\left(C_{j}: C_{i}\right)=.78$ and $P\left(C_{j}: I_{j}\right)=.66$; the first of these is a good approximation to the observed values for the $\mathrm{D}=8$ data in Table 3 , but the latter is much too low. In contrast, the serial processing model, with s taken equal to .13 for the $T_{2} T_{3}$ data,

TABLE III Test-retest Data $\left[\mathbf{P}\left(C_{j}: C_{i}\right)\right.$ and $\mathbf{P}\left(C_{j}: \mathbf{I}_{\mathbf{i}}\right)$

Denoting Proportion of Correct Detections on Test $\mathbf{T}_{\mathbf{j}}$

Given a Correct or an Incorrect Response,

Respectively, to the Same Display on Test $\mathbf{T}_{\mathbf{i}}$ ]

\begin{tabular}{ccccccc}
$\begin{array}{c}\text { Display } \\
\text { Size }\end{array}$ & $P\left(C_{2}: C_{1}\right)$ & $P\left(C_{2}: 1_{1}\right)$ & $P\left(C_{3}: C_{2}\right)$ & $P\left(C_{3}: 1_{1}\right) P\left(C_{3}: C_{1}\right) P\left(C_{3}: 1_{1}\right)$ \\
\hline 8 & .797 & .790 & .792 & .763 & .795 & .760 \\
12 & .744 & .652 & .789 & .624 & .778 & .652 \\
16 & .734 & .630 & .742 & .549 & .694 & .664 \\
\hline
\end{tabular}


TARLE IV Response Bias in Relation to Display Size

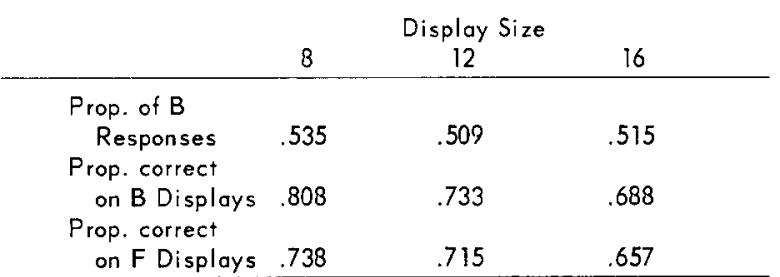

yields for theoretical values of $P\left(C_{j}: C_{i}\right)$ and $P\left(C_{j}: I_{j}\right)$ $\therefore .790$ and .744 at $\mathrm{D}=8 ; .742$ and .685 at $\mathrm{D}=12$; and .706 and .643 at $\mathrm{D}=16$.

Although mechanisms differing in some details from that of the serial processing model could account for the observed pattern, they must all have in common the property that the order in which the elements of the display are scanned is relatively constant and determined at least to a substantial extent by the positions of the elements in the display. Since the exposure time in the present study was too short to permit Ss to move their eyes and obtain more than one fixation of the presented material on any trial, this orderly scanning of the elements must be a central process, but whether its characteristics are determined entirely by the structures involved or are modifiable by learning remains a problem for future investigation.

Although not particularly germane to the hypotheses leading to the present experiment, data on response biases may be of some interest. In Table 4 are presented the proportions of trials on which Ss made $B$ responses (that is, reported $B$ rather than Fas a critical element), proportions of correct responses on trials when $B$ was the critical element in the display and proportions correct when $F$ was the critical element. The gist of these data is that there was a slight overall bias in favor of the $B$ over the $F$ response and a significant differential in correct responding to $B$ displays over $F$ displays. Since this disparity in correct responding to the two types of displays decreased with increasing display size, whereas the amount of guessing must have increased as a function of display size, it appears that the differential accuracy must have been due to differential perceptibility of the two cues rather than to a bias in guessing habits.

\section{Experiment II}

\section{Defection with Matrix Displays Containing Redundant Critical Elements}

In order to obtain somewhat more direct evidence concerning the degree of within-display correlation among responses to different elements, this experiment was designed with displays each of which contained only one of the two critical elements ( $B$ or F) but with the critical element appearing sometimes once and sometimes more than once in a display. It can be shown that, for any mean value of perceptual span (sample size), if there were no variability in sample size from trial to trial, accuracy of detection would be optimal (Estes, 1965). According to the serial processing model, detec- tion with displays containing redundant elements should be substantially less than optimal; the reason, in brief, is that on trials when the scanning process happens to continue until all or nearly all of the displayed elements have been scanned, $\mathrm{S}$ will gain little or no advantage from the presence of redundant critical elements. A number of alternative models that have been considered, including the differential perceptibility model discussed above, yield expectations of efficiency intermediate between the optimum and that prescribed by the serial processing model. It was possible conveniently to provide an additional source of information about the scanning process in this experiment by treating distance between redundant critical elements as a second independent variable.

\section{Method}

Apparatus. The experimental room, apparatus, and stimulus display cards were identical to those of the preceding experiment in all respects except for details of the displays which are described below.

Stimulus materials. All of the stimulus displays were arrays of 16 consonant letters arranged in 4 by 4 matrices according to the same format as that of the $D=16$ condition of the preceding experiment. Each display was composed of a number of randomly drawn "noise" consonants and either, 1,2 , or 4 instances of one of a pair of critical consonants ( $B$ and $F$ ). There were three sets of cards:

1. There were 32 cards containing but one instance of the critical letter; these were two parallel sets differing only in the member of the B/F pair that was present. In this set both the critical letter and each of the 15 filler letters appeared once in each of the 16 cells of the matrix.

2. There were 48 cards containing two instances of the critical letter; these were parallel sets of 24 each differing only in the member of the B/F pair that was represented. The critical letters did not appear in the 4 corner cells of the matrix; they appeared with equal frequency in the remaining 12 cells. On half of the cards, the two instances of the critical letters were in the same row or the same column (non-displaced condition); and were equally often separated by 0,1 , or 2 cells along the row or column. On the other half of the cards, the same conditions obtained except that at each separation, the critical elements were also displaced by one row or column. That is, if we number the cells from 1 to 4 in the top row of the display matrix, from 5 to 8 in the second row, etc., cards with 0,1 , and 2 separations in the non-displaced condition might have critical elements in cells 5 and 6,5 and 7,5 and 8 , respectively; then the corresponding cards in the displaced condition would have critical elements in cells 5 and 10, 5 and 11 , 5 and 12. In every case, one member of the pair appeared in one of the outer, or edge cells. The 14 filler letters were filled in randomly.

3. There were 32 cards containing four instances of the critical letter; these were two parallel sets of 16 each differing only in the member of the B/F pair that 
TABLE V Observed and Theoretical Proportions of Correct Detections

in Relation to Number of Critical Elements per Display

\begin{tabular}{|c|c|c|c|c|c|c|c|c|c|c|c|}
\hline & \multicolumn{5}{|c|}{ Parameter } & \multicolumn{5}{|c|}{ Number of Critical Elements } & \\
\hline & Esti & & & 1 & & & 2 & & & 4 & \\
\hline Subject & $\mathrm{p}$ & $d$ & Obs. & Ind. & FSSM & Obs. & Ind. & FSSM & Obs. & Ind. & FSSM \\
\hline 1 & .63 & 10 & .85 & .82 & .81 & .88 & .93 & .94 & 1.00 & .99 & 1.00 \\
\hline 2 & .66 & 10 & .81 & .83 & .81 & .96 & .94 & .94 & 1.00 & .99 & 1.00 \\
\hline 3 & .28 & 4 & .64 & .64 & .62 & .76 & .74 & .72 & .85 & .87 & .86 \\
\hline 4 & .51 & 8 & .75 & .76 & .75 & .90 & .88 & .88 & .94 & .97 & .98 \\
\hline 5 & .63 & 10 & .85 & .82 & .81 & .90 & .93 & .94 & .94 & .99 & 1.00 \\
\hline 6 & .04 & 1 & .52 & .52 & .53 & .51 & .54 & .56 & .60 & .58 & .62 \\
\hline 7 & .34 & 5 & .61 & .67 & .66 & .30 & .78 & .77 & .93 & .91 & .91 \\
\hline 8 & .58 & 9 & .79 & .79 & .78 & .91 & .91 & .91 & .98 & .98 & .99 \\
\hline Av. & & & .73 & .73 & .72 & .83 & .83 & .83 & .91 & .91 & .92 \\
\hline
\end{tabular}

was represented. The critical letters did not appear in either the 4 corner cells or the 4 central cells of the matrix. In one of the 2 cells of each of the remaining 4 pairs (one on each edge of the matrix) appeared one of the four cases of the critical letter. The set of these cards was exhaustive with respect to these groupings. The 12 filler letters were randomly inserted in the remaining cells.

Procedure. Ss were 8 Stanford students, paid for participation. After a $5 \mathrm{~min}$. allowance for adaptation to room brightness, each $S$ was seated at the apparatus and given a sample display with which to find a comfortable observing position and then to adjust the fixation spot. He was then instructed to report whether each display contained a " $B$ " or an " $F$ ", guessing whenever unsure. All the cards with 4 cases of the critical letter were now exposed, in a different random order for each S. A new stimulus exposure followed the response to the previous display by about $2 \mathrm{sec}$. Each exposure was preceded by a flash, $0.8 \mathrm{sec}$. in length, of a green light mounted at the bottom of the panel; $0.6 \mathrm{sec}$. intervened between the termination of this alerting signal and the stimulus exposure.

When the 4-case cards had all been exposed, the 2-case dịsplays were now shown, then the 1-case ones. At the conclusion of this series $S$ was given a 5 min. rest while the cards were reshuffled. The cards were now presented, with the same procedure, in the order 2-case, 1-case, 4-case. After another reshuffling, they were presented a third time, now in the order 1-case, 4-case, 2-case.

\section{Results}

Proportions of correct detections for each of the $8 \mathrm{Ss}$ on displays with 1,2 , or 4 critical element's are given in Table 5. The first question to be answered in connection with these data is whether the proportions of detections for 2 and 4 critical elements deviate significantly from the values that would be expected if detections of multiple critical cues were independent events. Under the latter hypothesis, if a common detection probability p obtains for all critical elements, then for a display with one critical element, probability of a correct report is

$$
P(C)=p+\frac{1-p}{2}=1-\frac{1}{2}(1-p)
$$

and in general, for a display with $m$ redundant critical elements,

$$
P(C)=1-\frac{1}{2}(1-p)^{m}
$$

The best fitting value of $\mathrm{p}$ was determined by least squares for each $S$, with the result shown in Table 5 . The standard error of the difference between observed and calculated values is less than .03 , and the 24 differences are half positive and half negative.

It might be noted that if the different critical elements in a display had different detection probabilities, relative efficiency of detection in 2 and 4 element displays should be greater than that predicted on the hypothesis of a common $p$ value. Suppose, for example, that the detection probabilities for two critical elements were $p_{1}$ and $P_{2}$ with $1 / 2\left(p_{1}+p_{2}\right)=p$. Then the probability of a correct report,

$$
P(C)=1-\frac{1}{2}\left(1-p_{1}\right)\left(1-P_{2}\right)
$$

would be greater than that given by Equation 5 with $\mathrm{m}=2$, for

$$
\left(1-p_{1}\right)\left(1-p_{2}\right) \leq(1-p)^{2}
$$

by the well-known theorem of the geometric and arithmetic means. An analogous result can be demonstrated for cases in which different detection probabilities are associated with different positions in the display (e.g., a value $p_{1}$ characterizing half of the cells in the display matrix and a value $p_{2}$ the other half) rather than with different critical elements. Evidently the data of the present experiment are quite compatible with the hypothesis that detections of multiple critical elements are independent events with a common p-value characterizing any given subject. On the other hand, as may be seen in the first column of Table 5, there are large individual differences in estimated p-values, so it would be hazardous to pool data over subjects for this type of analysis.

Taking these results together with those of Experiment 1 , we might seem to have a contradiction. For the independence hypothesis, which accounts admirably for the redundant-elements data, is quite incompatible with the test-retest data of Experiment 1, which exhibited substantial deviations from independence in the case of 
T.ABLE VI Mean Proportion of Correct Detections at each of Three Separations between Redundant Critical Elements, together with Theoretical Values for Fixed Sample Size Model

\begin{tabular}{cccc} 
Separation & Th. $(d=6)$ & Obs. & Th. $(d=7)$ \\
\hline 0 & .789 & .810 & .820 \\
1 & .844 & .844 & .891 \\
2 & .801 & .823 & .836 \\
\hline
\end{tabular}

12 and 16 element displays. We are thus led to inquire whether any of the models proposed for information processing in the forced-choice detection situation can accommodate the results of both experiments.

Considering first the serial processing model, we have evaluated the "stopping parameter" $s$ by least squares for the data of each subject in Table 5. The resulting correspondence of theoretical and observed values does not seem bad on casual inspection, but it is markedly inferior to that obtained on the independence hypothesis. Not only is the standard error of observed-vs.theoretical differences substantially larger for the serial processing model (greater than ${ }^{004}$ ), but also the detection probabilities for the 2 and 4 element displays are systematically underpredicted.

The fixed sample size model (Estes \& Taylor , 1964) comes off rather better. For any number m of redundant critical elements, probability of a correct response is

$$
P(C)=1-\frac{1}{2} \frac{\left(\begin{array}{c}
D-m \\
d
\end{array}\right)}{\left(\begin{array}{c}
D \\
d
\end{array}\right)}
$$

where $d$ is the sample size (i.e., the number of elements perceived on any trial from a display of size D). The parameter $d$ has been evaluated for each subject of the present experiment by least squares, as in the case of the other models, with the outcome exhibited in Table 5. The fit of the fixed sample size model to these data is much better than that of the serial processing model. One should note in this connection that the theoretical values for the fixed sample size model have been computed with $d$ estimated to the nearest integer only. By interpolation between neighboring integers the fit of the model could be further improved and would clearly be indistinguishable from that of the independence model. Discussion of this somewhat surprising outcome will be deferred until we treat the remaining empirical relationship under investigation in this experiment.

The other principal aspect of this experiment has to do with efficiency of detection as a function of distance between critical elements in a display. It will be recalled that in the 2 critical element displays, the two instances of B or F were separated by 0,1 , or 2 cells horizontally or vertically in the display matrix. For these three conditions, the observed mean proportions of correct detections were $.810, .844$, and .823 , respectively. This lack of any strong dependence of detection efficiency upon separation is rather at odds with one's intuitive expectation that the likelihood of perceiving at least one of the two critical elements should increase with the distance between them. However, to evaluate the implications of this result for the two main types of theoretical models under consideration we require some additional analysis.

Taking first the fixed sample size model, we note at once that if the elements perceived by the $S$ were sampled randomly from the entire display, we should expect probability of detection to be independent of distance between critical elements. If, however, the sampled elements must be adjacent, which seems more plausible on psychophysiological grounds, then it can be shown that probability of detection should increase with distance between critical elements, at least up to a point. An estimate of the degree of dependence predicted under the conditions of the present experiment can be obtained as follows.

On the basis of the data in Table 5, we assume that on the average 6-7 elements are effectively processed from a display of size 16. Further, we assume all samples of $k$ adjacent elements to be equally likely. There are various possible definitions of "adjacent" in this context; for calculational simplicity we take the following. Let the elements of the 4 by 4 matrix be numbered from left to right in each row, so that the top row comprises elements 1-4, the second row elements 5-8, and so on. Then any two elements numbered $n$ and $n+1$ are considered adjacent, with the additional proviso that element 16 is adjacent to element 1 . Now, if the 2 critical elements in a display occupy adjacent positions (separation $0)$, it is possible to draw a sample of 7 adjacent elements which contains neither of the critical elements in 8 different ways. If all 8 possible locations of the pair of critical elements and all 16 possible samples of 7 adjacent elements are equally likely, the probability that neither critical element is sampled is $\frac{8}{16}$. Similarly the probability of a "miss" is $7 / 16$ if the critical elements are separated by one space in any row, and $\frac{6}{16}$ if they are separated by two spaces. Probabilities of correct responses for the three cases would be then, $3 / 4,25 / 32$, and $13 / 16$. These results hold for the one-fourth of the display matrices in which the two critical elements were 0,1 , or 2 spaces apart in the same row. For the onefourth with the two critical elements 0,1 , or 2 columns apart in adjacent rows, the probabilities of correct responses can be similarly computed to be $7 / 8,29 / 32$, and $27 / 32$. Averaging the corresponding values for these and the other two types of displays, we obtain the theoretical values given for the fixed sample size model with $\mathrm{d}=7$ in Table 6 . In this same table are shown also the theoretical values for the same model with $d=6$ and the mean observed proportion of correct detections by the group of 8 subjects at each separation. Both the absolute level of correct responding and the pattern of differences as a function of separation appear compatible with the model.

A similar analysis in terms of the serial processing model, using the average value $s=.11$ from Table 5 , yields for the mean theoretical proportions of correct responses $.772, .792$, and .778 at separations of 0,1 and 
2, respectively. The predicted pattern of differences is not unpromising, but the predicted absolute level of correct responding is appreciably too low.

\section{Discussion}

The principal results of these two experiments may be summarized as follows:

1. The mean number of elements effectively processed increases with the number of elements in the display. The function relating number of elements processed to display size differs considerably from those prescribed either by a fixed sample size model or by one which assumes a probability distribution of probabilities-of-seeing over elements of area in the visual field; but it is compatible with the relation derivable from the serial processing model.

2. Test-retest results show relatively high correlations over widely separated $50 \mathrm{msec}$. exposures with respect to the particular elements sampled by a subject from a particular display.

3. The efficiency of detection with multiple, redundant critical elements conforms closely to expectation on the hypothesis of independence of response to different elements of a display. This result is compatible with the fixed sample size model but not with the serial processing model.

4. Probabilities of detection vary only slightly with respect to distance between critical elements in a display, highest efficiency being observed at the intermediate distance.

The pattern of findings appears somewhat paradoxical relative to the models under consideration. With the exception of test-retest relationships, all analyses involving only a single display size yield results which can be nicely interpreted, both qualitatively and quantitatively, in terms of the fixed sample size model. On the other hand, the observed function relating mean number of elements perceived to display size is quite unaccountable in terms of this model, whether one assumes sample size to be a constant number of elements or a constant proportion of the elements in the display.

The serial processing model handles test-retest phenomena and predicts the form of the display size function satisfactorily, but it predicts too much variance in number of elements processed from trial to trial. (For discussion of the relation between the large variance in sample size entailed by the serial processing model and the inferior account of the redundant elements data, see Estes, 1965). The aspect of the model primarily responsible for the large theoretical variance is the assumption that processing terminates on an all-or-none basis, all of the elements initially registered from a display being assumed available for processing up to some point in time and none thereafter. It would evidently be possible to change the variance predictions while leaving other salient properties of the model unaltered by modifying this one assumption. One alternative which seems intuitively plausible is to assume that the stimulus traces of the various elements in a display decay independently over time following exposure, but each according to the same exponential function which applies to the entire display as a unit in the present form of the model.

Although it has been useful in guiding the design of the present experiments and integrating the results, the serial processing model, even with revisions of the type indicated above, should not be taken as more than a provisional and incomplete prototype of a theory which may handle behavior involved in abstracting information from brief visual displays. Although our assumptions concerning the main features of the hypothesized scanning and categorizing processes appear to be well supported, there is considerable room for modification of these assumptions in quantitative details. Further elaboration of the model will be necessary before we can attempt quantitative accounts of such phenomena as figure-ground contrast (Eriksen, 1964) or visual afterimages (Teichner \& Wagner, 1964).

Our interpretation of behavior in the detection situation has involved no concepts of short-term memory or visual information storage (Sperling, 1963), unless the latter notion merely denotes the persistence of visual excitation beyond the termination of a display. This outcome is hardly surprising in view of the fact that our aim in developing the detection method was to minimize the role of memory in assessments of perceptual span. Assumptions concerning learning and memory will be required in a detailed theoretical account of the differences in estimates of perceptual span obtained with report and detection procedures, but discussion of these is beyond the scope of the present paper.

\section{References}

Averbach, E., \& Coriell, A. S. Short-term memory in vision. Bell tech. J., 1961. Pp. 309-328.

Broadbent, D. E. Perception and communication. New York: Pergamon Press, 1958.

Eriksen, C. W., \& Steffy, R. A. Short-term memory and retroactive interference in visual perception. J. exp. Psychol., 1964, 68, 423-434.

Estes, W. K. A technique for assessing variability of perceptual span. Proc. of the National Academy of Sciences, 1965, 53, No. $2,403-407$

Estes, W. K., \& Taylor, H. A. A detection method and probabilistic models for assessing information processing from brief visual displays. Proc. of the National Academy of Sciences, 1964, 52, No. 2., 446-454.

Sperling, G. The information available in brief visual presentations. Psychol. Monogr., 1960, 74, No. 11 (Whole No. 498), Sperling, G. A model for visual memory tasks. Hum. Factors, 1963, $5,19-32$.

Teichner, W. H., \& Wagner, M. Visual after-images as a source of information. Hum. Factors, 1964, 6, 141-156.

\section{Notes}

1. This research was supported in part by Grant G-24264 from the National Science Foundation and Grant $M H-6154$ from the United States Public Health Service. The first author is responsible for the theoretical analyses and for the present report. The second author participated in the planning and conducting of both experiments.

2. Built by Iconix, Inc., of Menlo Park, California

3. Rise and decay times for light intensity output have been described in a previous report (Estes \& Taylor, 1964).

4. For simplicity, the parameter $m$ in Equation 1 has been taken equal to zero for all theoretical calculations given in this paper. 